\title{
Local tumor irradiation augments the antitumor effect of cytokine-producing autologous cancer cell vaccines in a murine glioma model
}

\author{
Katalin Lumniczky, ${ }^{1}$ Szilvia Desaknai, ${ }^{1}$ Laszlo Mangel, ${ }^{2}$ Bela Szende, ${ }^{3}$ Hirofumi Hamada, \\ Egon J Hidvegi, 1 and Geza Safrany ${ }^{1}$ \\ ${ }^{1}$ Department of Molecular and Tumor Radiobiology, National Research Institute for Radiobiology and \\ Radiohygiene, Budapest, Hungary; ${ }^{2}$ Department of Radiation Therapy, National Institute for Oncology, Budapest, \\ Hungary; ${ }^{3}$ First Institute of Pathology and Experimental Cancer Research, Semmelweis University, Budapest, \\ Hungary; and ${ }^{4}$ Department of Molecular Medicine, Biomedical Research Center, Sapporo Medical University, \\ Sapporo, Japan.
}

\begin{abstract}
The combined therapeutic effect of cytokine-producing cancer cell vaccines and local radiotherapy was studied in a mouse glioma 261 (Gl261) brain tumor model. Brain tumor-bearing mice were treated with cytokine (IL - 4, IL-6, IL - 7, GM-CSF, TNF- $\alpha$, LIF, LT) producing vaccines made by in vitro transduction of Gl261 cells with the corresponding adenoviral vectors. Vaccines producing either IL - 4 or GM-CSF cured $20-40 \%$ of mice. The antitumor effect strongly depended on the secreted cytokine level. Vaccination therapy induced specific activation of cytotoxic T lymphocytes measured by cell-mediated cytotoxicity assay. Brain tumors were heavily infiltrated by $\mathrm{CD}_{4}{ }^{+}$lymphocytes after treatment with IL-4- or GM-CSF-secreting cells. GM-CSF vaccination induced moderate $\mathrm{CD}^{+}$infiltration, as well. Depleting either $\mathrm{CD}^{+}{ }^{+}$or $\mathrm{CD}^{+}$lymphocyte subsets abolished the anticancer effect of $\mathrm{GM}$ CSF-expressing cells. Strong synergism was observed by combining cytokine vaccination (GM-CSF, IL - 4, IL-12) with local tumor irradiation: about $80-100 \%$ of the glioma-bearing mice was cured. The high efficiency of combined treatment was maintained even under suboptimal conditions when neither of the modalities cured any of the mice alone. This suggests that vaccination therapy might open a new potential in the clinical treatment of high-grade gliomas when applied as adjuvant to existing treatment modalities.
\end{abstract}

Cancer Gene Therapy (2002) 9, 44-52 DOI: 10.1038/sj/cgt/7700398

Keywords: GM-CSF; IL -4; IL -12; antiglioma vaccine; irradiation

$I^{n}$ humans, gliomas constitute about $30-45 \%$ of all intracranial tumors. Glioblastoma multiforme is the most frequent form of glioma and is characterized by very rapid local growth and invasiveness. Gliomas do not metastasize, but their local growth is almost always fatal. Recent advances in neurosurgical techniques, radiation therapy, and chemotherapy have failed to improve substantially the prognosis of this malignant tumor. The life expectancy of patients with glioblastoma is usually less than 1 year from the time of diagnosis and the 5-year survival rate is only about $5 \%{ }^{1,2}$ Therefore, it is necessary to develop new therapeutic modalities.

There are several immune therapy approaches that might increase the immunogenicity of the tumors. ${ }^{3,4}$ One possibility is the introduction of cytokine-encoding genes into the tumor cells. This can be achieved either by direct intratumoral injection of viral vectors or by ex vivo modification

Received August 29, 2001.

Address correspondence and reprint requests to: Dr Geza Safrany, Department of Molecular and Tumor Radiobiology, National Research Institute for Radiobiology and Radiohygiene, Anna u. 5, Budapest H-1221, Hungary.E-mail: safrany@hp.osski.hu of the malignant cells. The direct intratumoral vector injection is much simpler than the ex vivo modification, but there are certain risks arising from the introduction of a large number of viral particles into the human body. During the ex vivo approach, most of the tumor is removed by surgery and a primary cell culture is established from the malignant tissue. Then cytokine-encoding vectors are introduced into the in vitro growing tumor cells and cell division is stopped by high-dose irradiation of the culture. Finally, the cytokine-expressing irradiated tumor cells are used to vaccinate the same patient from whom the original tumor was removed. It is expected that the host immune system is activated by the vaccine, and it will attack both the residual tumor cells at the site of the surgery and the cells at distant metastases. The key requirement of this protocol is the presentation of tumor-associated antigens in the microenvironment of cytokine secretion. 5,6

The central nervous system (CNS) has long been considered as an immunologically privileged site. Several factors may contribute to this status, including an endothelial structure that restricts passage of macromolecules, viruses, and cells, the absence of lymphatic drainage, and finally the inadequate expression of MHC molecules. 
Despite difficulties generating an adequate immune response in the $\mathrm{CNS}$, there are evidences that $\mathrm{T}$ lymphocytes can penetrate the blood-brain barrier and mediate an efficient tumor growth inhibitory effect. ${ }^{7}$

Formerly, we studied the therapeutic effect of autologous cancer cell vaccines producing either IL-2 or IL-12 in the mouse G1261 brain tumor model. Both vaccines exhibited antitumor effects: about $30-40 \%$ of mice was cured. In both cases, the therapeutic effect of the vaccines strongly depended on the expressed cytokine level. ${ }^{8}$ In the present paper, we report the anticancer effect of GM-CSF- and/or IL-4-secreting glioma vaccines. The novel therapeutic approaches should be used in combination with existing treatment modalities. Therefore, special emphasis was put on the combined antiglioma effect of cytokine vaccination and local tumor irradiation.

\section{Materials and methods}

\section{Cell lines, recombinant adenoviruses}

Hybridoma cell lines GK1.5 and 53-6.72, secreting monoclonal antibodies against $\mathrm{CD}^{+}$and $\mathrm{CD}^{+}$lymphocyte subsets, were bought from ATCC (Manassas, VA, USA) and maintained according to the supplier's suggestion. The G1261 mouse glioma tumor was originally induced by intracerebral injection of a chemical carcinogen into $\mathrm{C} 57 \mathrm{Bl} /$ 6 mice $^{9}$ and maintained on its syngeneic host by alternating intracranial and subcutaneous implantation of small tumor pieces. We have established a permanent cell line (G1261) from the tumor. The cells were maintained in DMEM culture medium supplemented by $10 \%$ fetal calf serum. Detailed characterization of the cell line will be published elsewhere.

The adenoviral vectors AdexCAmIL-2, AdexCAmIL-4, AdexCAmIL -6, AdexCAmIL - 7, AdexCAmIL-12p35, AdexCAmIL-12p40, AdexCAmGM-CSF, AdexCAmLT, AdexCAmLIF, and AdexCAmTNF - $\alpha$ encoding the corresponding cytokines were created and very generously provided by Dr. Hamada. ${ }^{10}$ Note that the two subunits of the mouse IL-12 gene (p35 and p40) were present in two different constructs. During vector creation, the E1A, E1B, and E3 regions of adenovirus type 5 were removed, rendering the vector replication incompetent in most mammalian cells. The E1A-E1B region was replaced with a cloning cassette containing the appropriate cytokine gene. Cytokine expression was driven by an artificial promoter composed of the core promoter region of the chicken $\beta$-actin gene and of the enhancer region of the major early promoter of the cytomegalovirus. Transformed human embryonic kidney 293 cells (ATCC) were used for vector propagation and virus isolation. ${ }^{10}$

Brain tumor model and treatment by cytokine-secreting autologous mouse glioma vaccines and/or by radiation therapy

Animal studies were done according to Hungarian national regulations. Logarithmically growing G1261 cells were harvested and suspended in Hank's balanced salt solution. Brain tumors were induced by intracranial injection of 1$2 \times 10^{4}$ Gl261 cells in $10-\mu \mathrm{L}$ final volume. Cells were implanted through Fissura petrosquamosa into the right cerebral hemisphere of anesthesized 8 - to 12 -week-old $\mathrm{C} 57 \mathrm{Bl} / 6$ mice at $4 \mathrm{~mm}$ depth below the surface of the scull using a $250-\mu \mathrm{L}$ Hamilton syringe. When radiation therapy was combined with GM-CSF vaccination, $1 \times 10^{5} \mathrm{Gl} 261$ cells were transplanted.

Vaccination with cytokine-producing cells was performed 3 days after tumor transplantation. To create the cytokineproducing vaccines, in vitro growing G1261 cells were transduced at different multiplicity of infection (MOI) with the corresponding adenoviral vectors. ${ }^{10}$ Cytokine production was measured in the culture supernatant 48 hours later by conventional ELISA kits (Endogen, Woburn, MA, USA and BioSource Europe, Nivelles, Belgium) and cells were irradiated with 20 Gy ${ }^{60} \mathrm{Co}-\gamma$ radiation (Gammatron-3 radiation source, dose rate: $0.4781 \mathrm{~Gy} / \mathrm{min}$; Siemens, Erlangen, Germany) to stop cell division. Cells were harvested by trypsin treatment and $1 \times 10^{6}$ cells were injected subcutaneously to vaccinate tumor-bearing mice. Mice were killed when they were moribund or all mice were killed 100 days after tumor induction. According to our experience, if a mouse survived for 100 days, it was completely tumorfree for at least a year. All mice were carefully autopsied. At least three independent experiments including five to seven mice were performed for each treatment.

For local radiotherapy, the head of anesthesized mice was irradiated with 6 Gy $x$-ray radiation (THX-250 Therapeutic $\mathrm{x}$-ray SOURCE, dose rate: $1.003 \mathrm{~Gy} / \mathrm{min}$; Medicor, Budapest, Hungary) 3 days after tumor transplantation. A lead tube covered the other part of the body to protect it from radiation. For the combined modality treatment, vaccination with cytokine-producing cells was performed 1 hour after irradiation.

Statistical analysis was done by Mantel-Cox test. A $P$ value of $<.02$ was considered significant.

Monoclonal antibody production and depletion of $\mathrm{CD}^{+}$ and $C D 8^{+}$lymphocyte subsets

Mouse hybridoma 53-6.72 cells were maintained under in vitro conditions as recommended by ATCC and anti-

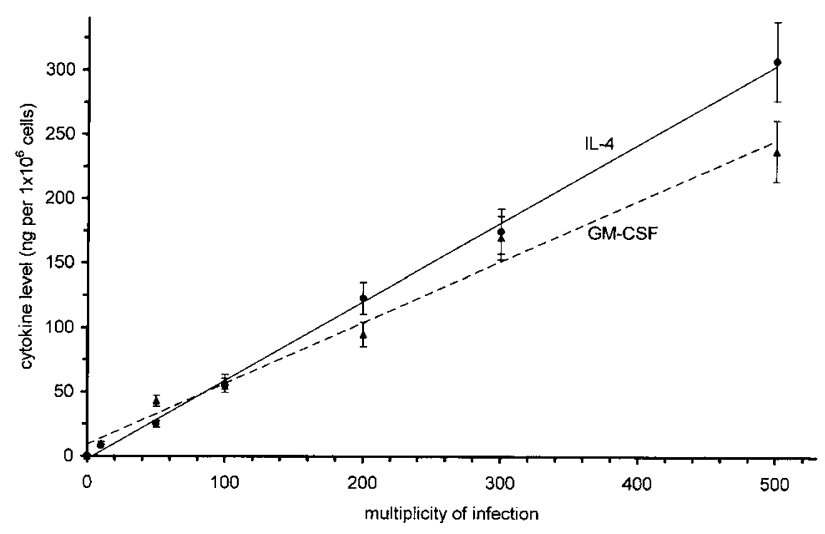

Figure 1 Cytokine levels secreted by gene-modified GI261 cells. In vitro growing Gl261 cells were transduced at different $\mathrm{MOI}$ by adenoviral vectors encoding either IL-4 or GM-CSF. Cytokine concentration was measured in the culture supernatant 24 hours after transduction by conventional ELISA kits. 
46
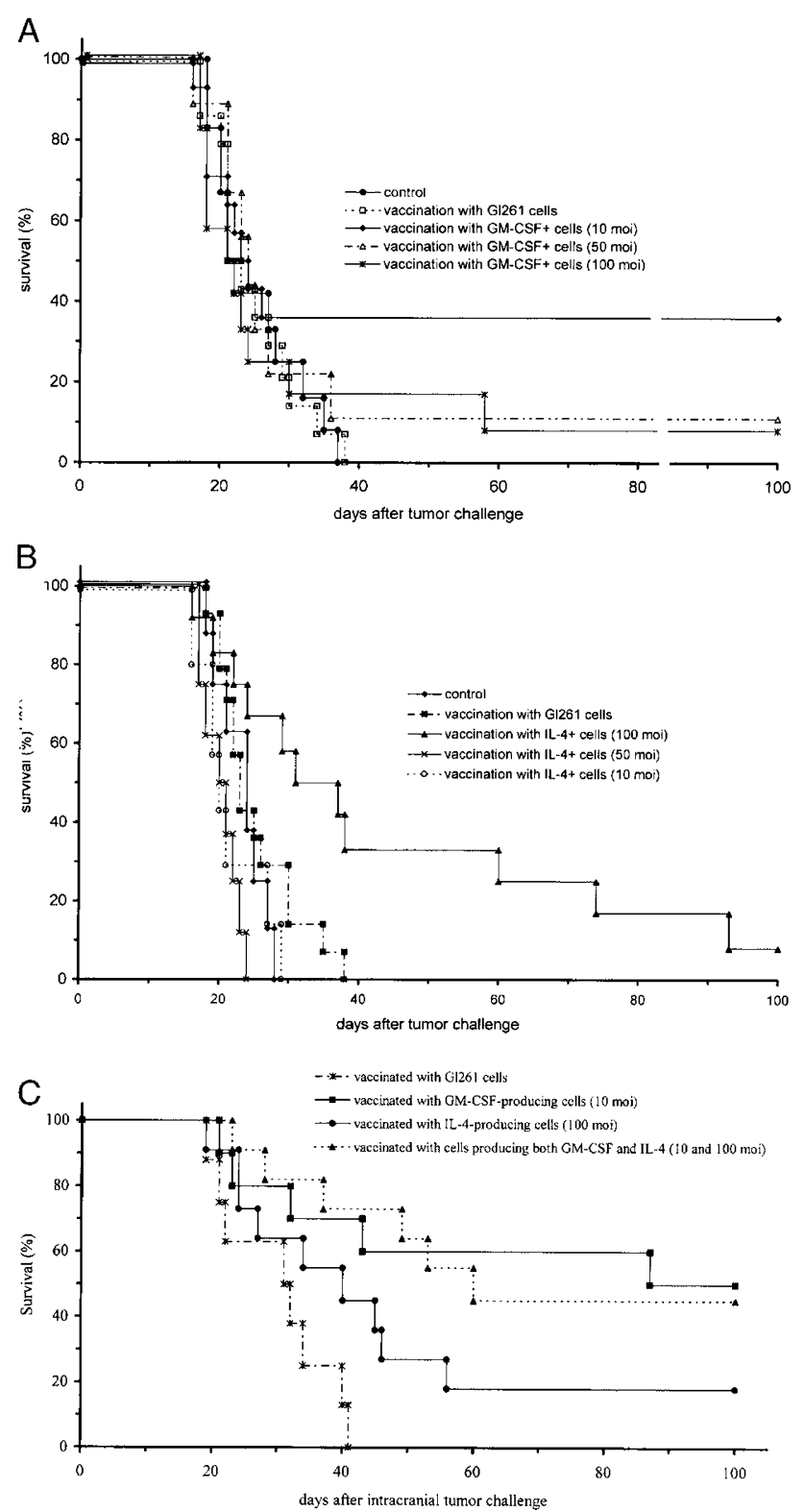

Figure 2 Brain tumor treatment by cytokine-secreting autologous cancer cells improves survival. Glioma-bearing mice were treated with cytokine-secreting vaccines 3 days after intracranial transplantation of Gl261 cells. For vaccine preparation, in vitro growing GI261 cells were transduced at the indicated MOI by adenoviral vectors encoding the corresponding cytokines. Cells were irradiated and harvested 48 hours later and $1 \times 10^{6}$ cells were used for subcutaneous vaccination of brain tumor-bearing mice. A: Treatment by GM-CSF-secreting vaccines. $P<.008$ for $10 \mathrm{MOI} G M-$ CSF vaccine versus the GI261 vaccine. B: Vaccination by IL-4producing cells. $P<.02$ for $100 \mathrm{MOI}$ IL -4 vaccine versus the Gl261 vaccine. C: Treatment by a vaccine secreting both IL-4 and GMCSF. $P<.001,<.016$, and $<.0008$ for the GM-CSF, IL-4, and dual cytokine-secreting vaccines versus the Gl261 vaccine, respectively.

$\mathrm{CD}^{+}$antibody was purified by DE52 (Whatman, Springfield, UK) ion exchange chromatography as described. ${ }^{11}$ To isolate anti-CD4 ${ }^{+}$antibodies, GK1.5 cells were intraperitoneally injected into nude mice and the collected ascites fluid was directly used after appropriate dilution. ${ }^{11}$

The $\mathrm{CD}^{+}$and $\mathrm{CD} 8^{+}$lymphocyte subsets were depleted by treating $\mathrm{C} 57 \mathrm{Bl} / 6$ mice with corresponding antibodies. ${ }^{11}$ After pretreatment with monoclonal antibodies, brain tumors were induced and vaccination with GM-CSF-expressing cells was performed as described above.

\section{Cell-mediated cytotoxicity assay}

Healthy mice were vaccinated with either irradiated G1261 cells or with mIL-4- or mGM-CSF-producing G1261 cells at therapeutic concentrations (see below). The spleens were collected at different intervals after vaccination (3 days, 1, 2 , and 3 weeks) and lymphocytes isolated. The lymphocytes were preincubated in the presence of $\mathrm{IL}-2$ with irradiated Gl261 cells for 5 days and then mixed with ${ }^{51} \mathrm{Cr}$ labelled G1261 or B16 melanoma cells at different effectorto-target ratios. The amount of radioactivity released in the medium was used for the evaluation of lymphocyte activation. $^{12}$

\section{Immunohistochemistry}

Brain tumor-bearing mice were vaccinated with IL-2-, IL4-, IL-12-, and GM-CSF-secreting irradiated Gl261 cells. Mice were killed and brain tumors removed 10 days after vaccination. The tumors were fixed in $4 \%$ buffered formaldehyde, embedded in paraffin, and $8-\mu \mathrm{m}$ thin sections were cut. Sections were routinely stained in H\&E. Immunohistochemical reactions ${ }^{13}$ were performed on fixed sections using polyclonal antibodies directed against $\mathrm{CD} 4^{+}$ and $\mathrm{CD}^{+}$cells $(\mathrm{Sc}-7219$ and $\mathrm{Sc}-7188$; Santa Cruz Biotechnology, Santa Cruz, CA). The primary antibodies were used in 1:100 dilutions. Diaminobenzidine (DAB) was applied to visualize the reaction. Normal mouse lymph node was used as positive control. Negative controls were

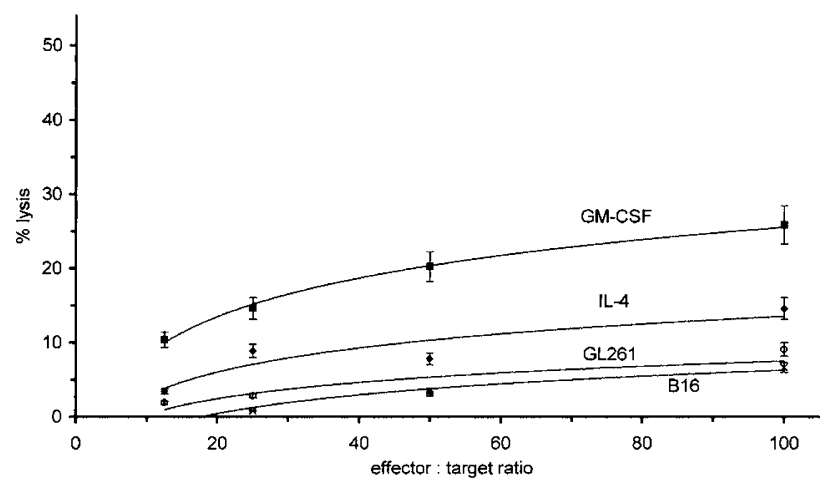

Figure 3 Induction of CTL in mice after vaccination by cytokineexpressing autologous cancer cells. Healthy mice were vaccinated either by irradiated GI261 or by IL -4- or GM-CSF-secreting GI261 cells. CTL activity, against ${ }^{51} \mathrm{Cr}$-labeled Gl261 cells was measured 3 weeks after vaccination, as described in Materials and methods. In the experiment marked with B16, vaccination was performed with GM-CSF-secreting cells, but the targets were ${ }^{51} \mathrm{Cr}$-labeled murine B16 melanoma cells. 

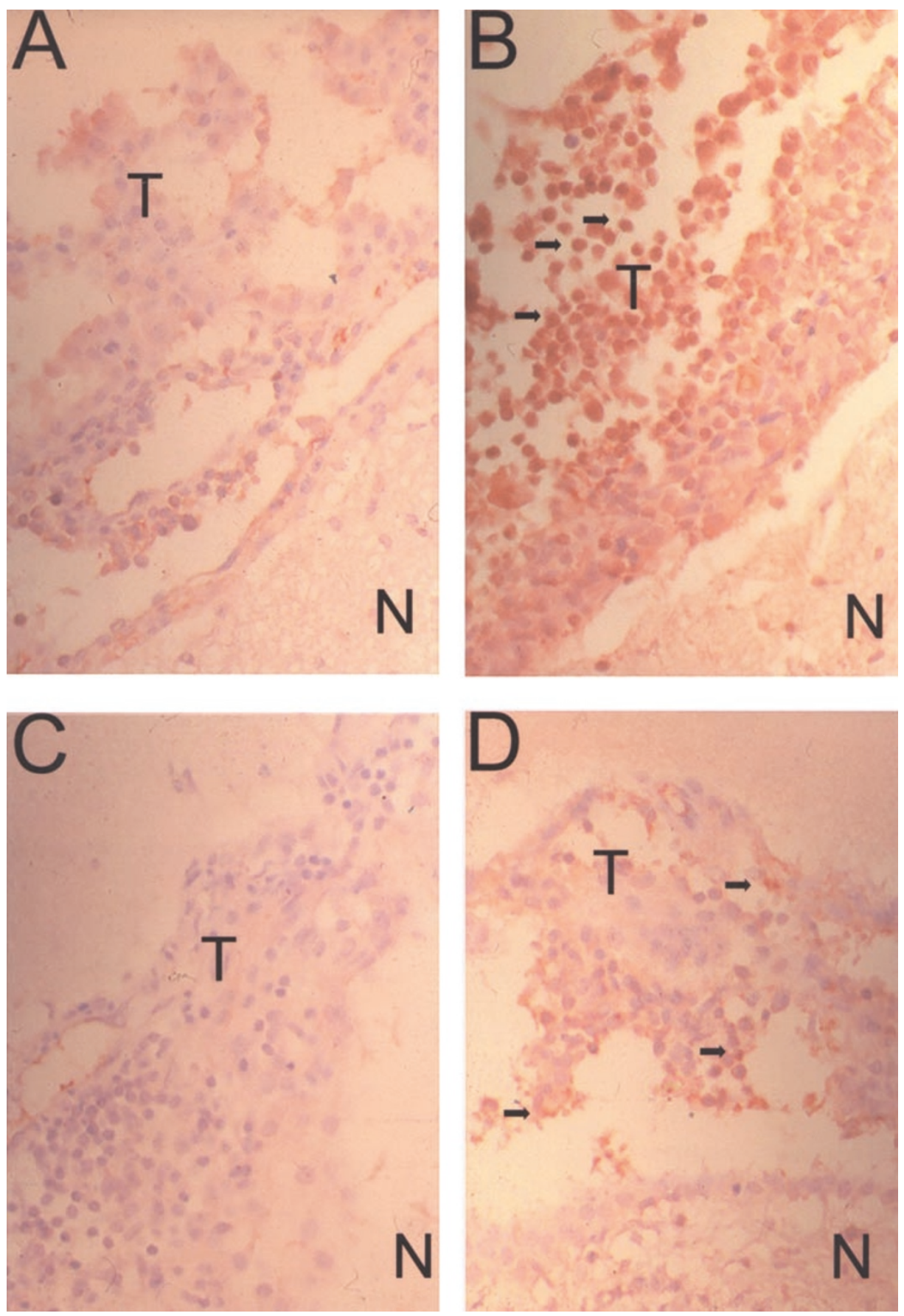

Figure 4 Immune histochemistry of brain tumors after treatment with GM-CSFexpressing cancer cells. Brain tumorbearing mice were vaccinated either with GI261 cells (Panels A and C) or GM CSF-secreting Gl261 cells (Panels B and D). Tumor infiltration by $\mathrm{CD}^{+}$ (Panels A and B) and CD8 ${ }^{+}$(Panels C and D) lymphocyte subsets was investigated 10 days after vaccination by immune histochemistry as described in Materials and methods. $\mathrm{N}$, normal brain tissue; $\mathrm{T}$, brain tumor. The arrows show the tumor infiltrating $\mathrm{CD}^{+}{ }^{+}$and $\mathrm{CD}^{+}$lymphocytes, respectively. achieved by omitting the primary antibody. Light green was applied as counterstain.

\section{Results}

Transduction efficiency and cytokine production in GI261 cells

First, the transduction efficiency of adenoviral vectors was investigated in G1261 cells. A construct (Adex1CAlacZ) encoding the bacterial lac $Z$ gene was introduced into in vitro growing Gl261 cells at different MOI and the presence of lac $Z$ activity measured. ${ }^{10}$ At ten MOI, about $50-70 \%$ of the cells was transduced. The transduction efficiency increased linearly up to $100 \mathrm{MOI}$ when all of the cells were already infected. ${ }^{8}$ This proved that adenoviral vectors could transduce Gl261 cells with high efficiency.

Next, cytokine-encoding adenoviral vectors (AdexCAmGM-CSF, AdexCAmIL-4) were introduced into G1261 cells at different MOI and cytokine expression was measured in the culture medium by conventional ELISA kits. Both GM-CSF and IL-4 levels increased linearly in the medium at least up to $500 \mathrm{MOI}$ (Fig 1). As mentioned before, at $100 \mathrm{MOI}$, the vector already infected 
all of the cells. The linear increase in the cytokine level beyond 100 MOI suggests that the cells were infected not only by one, but also by several virus particles. The data also show that altering the MOI during transduction could very easily modify the cytokine production of the cancer cell vaccines.

\section{Tumor growth inhibition by cytokine-producing} autologous glioma cell vaccines

The therapeutic effect of cytokine-producing autologous cancer cell vaccines was investigated in a mouse glioma 261 brain tumor model. Brain tumors were transplanted by intracranial injection of $1-2 \times 10^{4}$ G1261 cells. Three days later, tumor-bearing mice were vaccinated with G1261 cells expressing various levels of different cytokines. For vaccine preparation, in vitro growing G1261 cells were transduced at different MOI with adenoviral vectors encoding various cytokines (IL-4, IL-6, IL-7, GM-CSF, LIF, LT, and TNF- $\alpha$ ). Two days later, cytokine-producing cells were irradiated, harvested, and used for subcutaneous vaccination of brain tumor-bearing mice.

The therapeutic effect of the vaccines strongly depended both on the type and on the level of the secreted cytokine. Vaccines expressing IL-6, IL-7, LIF, LT, and TNF - $\alpha$ were inefficient to prolong the survival of glioma-bearing mice when produced by transduction at 10, 50, 100, or 200 MOI (data not shown). However, vaccines producing either GM-CSF or IL-4 cured a substantial portion of the mice (Fig 2A and B). In these cases, the survival rate depended very strongly on the produced cytokine level. For the GM-CSF-expressing vaccine, the highest survival rate $(30-40 \%$; Figure $2 \mathrm{~A}$ ) was obtained when 10 MOI was used for transduction during vaccine preparation. This vaccine produced about $10 \mathrm{ng}$ of GM-CSF $/ 1 \times 10^{6}$ cells $/ 24 \mathrm{~h}$ (Fig 1 ). Vaccines, secreting higher levels of GM-CSF (50 or 100 MOI), were much less efficient (Fig 2A).

IL-4-secreting vaccines were effective, if cells were transduced at $100 \mathrm{MOI}$ with the adenoviral vector. This vaccine expressed about $50 \mathrm{ng}$ of IL- $4 / 1 \times 10^{6}$ cells $/ 24 \mathrm{~h}$ (Fig 1 ) and prolonged the lifespan and/or cured a significant portion of mice (Fig 2B). Vaccines produced at lower MOI were inefficient.

Because both IL-4- and GM-CSF-producing vaccines were able to cure mice, we studied the combined effect of these two vaccines. Glioma-bearing mice were treated with G1261 cells that were co-transduced with IL-4 (100 MOI) and GM-CSF (10 MOI) encoding adenoviral vectors. The therapeutic effect of the double cytokinesecreting vaccine was similar to the GM-CSF-producing vaccine (Fig 2C).

Vaccination by cytokine-producing cells activates cell-mediated antitumor immune response

Three lines of experiments were performed to study the activation of the immune system against the tumor after vaccination with cytokine-producing G1261 cells. First, we investigated the activation of cytotoxic $\mathrm{T}$ lymphocytes (CTL) against glioma cells. Healthy mice were vaccinated with IL-4- or GM-CSF-expressing G1261 cells at therapeutic cytokine concentrations as described above. Mice were killed at different intervals after vaccination and lymphocytes were prepared from the spleen. The CTL response was measured as described in Materials and methods. CTL activation was not detected during the first week, and then it gradually increased and peaked 3 weeks after vaccination (Fig 3). The CTL response was much higher for the GM-CSF-producing vaccine than for the IL4-secreting vaccine.

The involvement of $\mathrm{CD}^{+}$and $\mathrm{CD}^{+}$lymphocyte subsets in the antitumor response has been also studied. Glioma-bearing mice were vaccinated with IL-2-, IL-4-, IL-12-, or GM-CSF-expressing cells. The controls were vaccinated with irradiated G1261 cells. Mice were killed 10 days later, brain tumors removed, and the infiltration of tumors by $\mathrm{CD}^{+}$and $\mathrm{CD}^{+}$lymphocyte subsets was investigated by immune histochemistry. When the tumorbearing mice were treated with GM-CSF-producing cells, the brain tumors were heavily infiltrated by $\mathrm{CD}^{+}$ lymphocytes (Fig 4A and B). Similar data have been obtained with IL-2-, IL-4-, and IL-12-secreting cells (data not shown). The GM-CSF-producing vaccine induced moderate tumor infiltration by $\mathrm{CD} 8{ }^{+}$lymphocytes (Fig 4C and D), as well. CD8 ${ }^{+}$infiltration was not detected with other vaccines (not shown).

The role of the $\mathrm{CD}^{+}{ }^{+}$and $\mathrm{CD}^{+}$lymphocytes in the antitumor response was further investigated by depleting these cells before tumor induction. Healthy mice were pretreated with antibodies against either $\mathrm{CD}^{+}$or $\mathrm{CD}^{+}$ lymphocyte subsets. ${ }^{11}$ Brain tumors were induced by intracranial injection of G1261 cells and 3 days later, glioma-bearing mice were vaccinated with GM-CSFproducing autologous cancer cells. Depletion of either $\mathrm{CD}^{+}$or $\mathrm{CD}^{+}$lymphocytes equally prevented the antitumor effect of the vaccine (Fig 5 ).

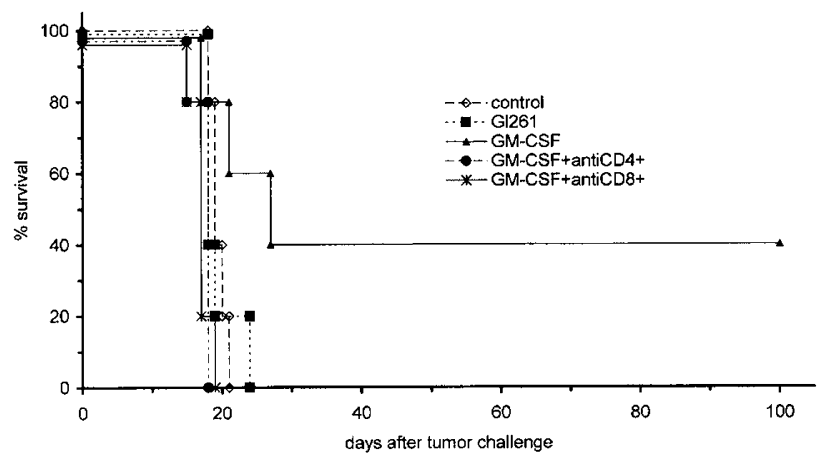

Figure 5 Depletion of $\mathrm{CD}^{+}$and $\mathrm{CD}^{+}$lymphocytes eliminates the tumor growth-inhibitory effect of GM-CSF-producing vaccines. Glioma-bearing mice were vaccinated with autologous cancer cells transduced at $10 \mathrm{MOI}$ by AdexCAmGM-CSF. In experiments labeled as GM-CSF+anti-CD4 ${ }^{+}$and GM-CSF+anti-CD8 ${ }^{+}$, the corresponding lymphocyte subsets were eliminated by pretreatment with appropriate monoclonal antibodies before tumor transplantation. $P<.006$ for $10 \mathrm{MOI}$ GM-CSF vaccine versus the GI261 vaccine. 
Combination of antiglioma vaccination with local tumor irradiation considerably improves survival

As mentioned above, vaccination with certain cytokineexpressing autologous cancer cells was able to cure about $20-40 \%$ of glioma-bearing mice (Fig 2). We have investigated whether the combination of the vaccination protocol with local radiation therapy could further improve survival. The tumor was induced by intracranial transplantation of $1-2 \times 10^{4}$ G1261 cells. Three days later, the head of the glioma-bearing mice was irradiated with $6 \mathrm{~Gy}$ $\mathrm{x}$-ray radiation. One hour after irradiation, mice were
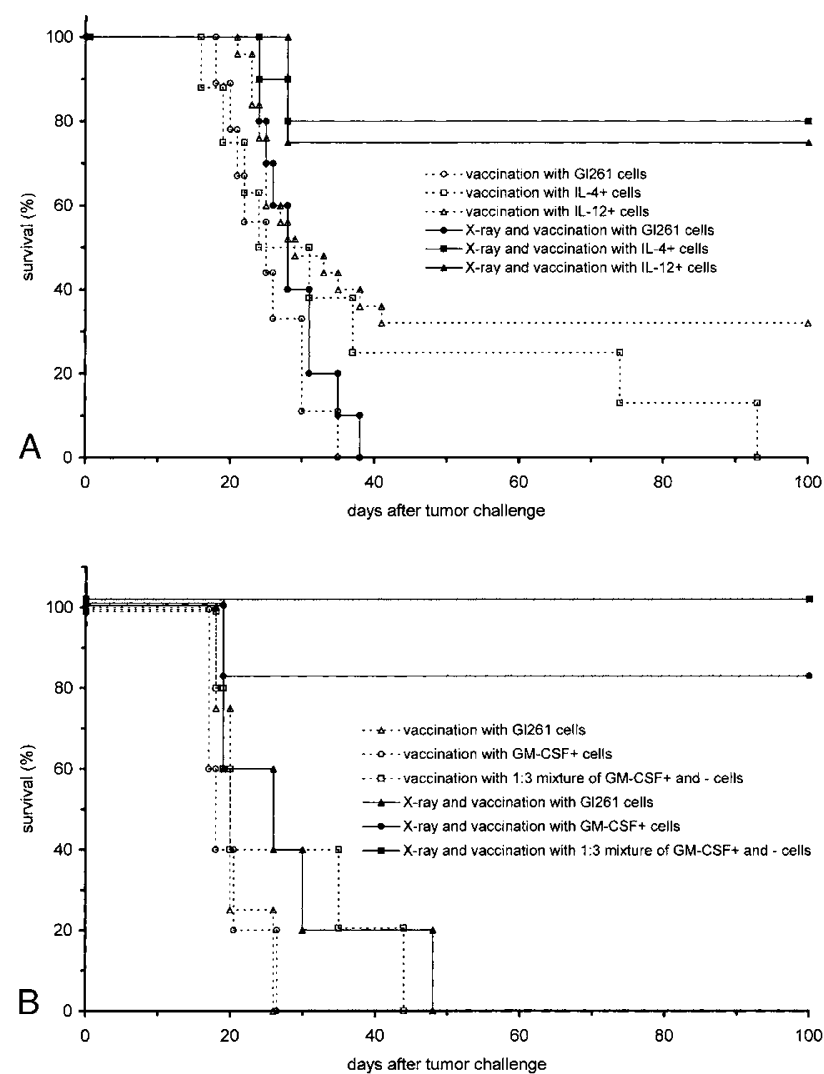

Figure 6 Combination of local tumor irradiation with vaccination by cytokine-producing cancer cells improves survival. A: Vaccination with IL-4- or IL-12-producing cells. Brain tumors were induced by intracranial transplantation of $1 \times 10^{4}$ Gl261 cells. Glioma-bearing mice were vaccinated with IL-12- or IL-4-secreting cells and/or irradiated with $6 \mathrm{~Gy} x$-ray radiations. $P<.01$ and $<.02$ for the $\mathrm{IL}-12$ and IL -4 vaccines versus the GI261 vaccine. $P<.000$ and $<.000$ for the combined treatments with irradiation and vaccination with IL - 12or IL-4-secreting cells versus the Gl261 - vaccinated and -irradiated group, respectively. B: Vaccination with GM-CSF-secreting cells. Brain tumors were induced by intracranial transplantation of $1 \times 10^{5}$ GI261 cells. Mice were treated with GI261 cells, with GM-CSFproducing GI261 cells transduced at $200 \mathrm{MOI}$ and with the 1:3 mixtures of transduced and nontransduced cells, respectively. The combined modality groups were vaccinated as shown, and local irradiation was performed with 6 Gy $x$-ray. $P<.002$ for the combined treatment with radiation and vaccination with $\mathrm{GM}-\mathrm{CSF}$-secreting cells and $<.000$ for the treatment with radiation and vaccination with 1:3 mixture of GM-CSF-secreting and nonproducing cells versus the GI261 - vaccinated and - irradiated group, respectively. vaccinated with either IL-4- or IL-12-producing ${ }^{8}$ G1261 cells at therapeutic cytokine concentrations. The combination of radiotherapy with vaccination by cancer cells secreting either IL-4 or IL-12 was very efficient. The tumor was completely eliminated in about $80 \%$ of glioma-bearing mice by the combined modality treatments (Fig 6A).

To investigate the limits of the combined protocol, brain tumor was induced by 10 -fold higher cell number $\left(1 \times 10^{5}\right)$ as usual. This cell dosage killed the untreated mice very rapidly. Three days later, the head of the mice was irradiated as mentioned before. Within 1 hour, mice were vaccinated with GM-CSF-expressing G1261 cells transduced at 200 MOI. Vaccination alone with cells expressing such a high level of GM-CSF has not improved survival rate at all (Fig 6B). To slightly reduce the secreted cytokine level, GM-CSF-producing cells were mixed in 1:3 ratios with nontransduced cells. Vaccination with this mixture moderately improved lifespan, but none of the mice was cured of the tumor. Radiation alone slightly increased survival rates, but again, none of the mice survived. However, the combination of vaccination therapy with local irradiation considerably enhanced survival. About $80 \%$ of the mice was completely cured of the tumor if they were irradiated and vaccinated with cells secreting high levels of GMCSF. When the secreted GM-CSF levels were slightly lower (1:3 mixtures of GM-CSF transduced and nontransduced cells), all of the mice recovered from the brain tumor (Fig 6B).

\section{Discussion}

High-grade gliomas belong to the most aggressive malignant diseases with very poor prognosis. The induction of the immune system against cancer cells might open new potentials in the treatment of brain tumors. ${ }^{14}$ Recently, the potential tumor growth inhibitory effect of various immunostimulatory protocols has been studied by a number of investigators in intracranial tumor models. The models included intracerebrally implanted murine melanomas, ${ }^{15-17}$ as well as rat $^{18-26}$ and murine glioma cells. ${ }^{27-32}$ The immunostimulatory genes were delivered by several vehicles, including retroviral vectors, vaccinia virus, plasmids, and liposomes. Interestingly, adenoviral vectors were rarely used for this purpose. ${ }^{33}$ Our data showed that the advantage of the adenoviral vector system is its high transduction efficiency. At rather low MOI, both murine G1261 cells, as well as primary human glioma cells (latter is not shown), could be transduced with $100 \%$ efficiency by adenoviral vectors. The other benefit of the adenoviral vector system is that at high MOI, the transduced cells may contain many viral particles. The cytokine level produced by the vaccine is linearly related to the number of viral particles per cell or to the applied MOI. With adenoviral vectors, it is easy to alter the cytokine level secreted by the gene-modified cancer cells.

A high number of cytokine genes (IL-2, IL - 4, IL - 6, IL - 7, IL - 12, GM-CSF, LIF, LT, and TNF- $\alpha$ ) have been used by 
us for the genetic modification of the ex vivo growing tumor cells. Others have not tested such a high panel of cytokines against gliomas. We have found that from the wide panel of cytokines, only IL-2-, ${ }^{8}$ IL-4-, IL-12-, and GM-CSF-secreting vaccines prolonged lifetime and cured substantial proportion of glioma-bearing mice. This is in good agreement with previous findings that GM-CSF, ${ }^{15-17,28,30}$ IL-4, ${ }^{15,18,19,22,25}$ IL- $12,{ }^{21,24}$ or IL$2^{21,23,3133}$ production by cancer cells is in association with prolonged antitumor effect.

Our data suggested that the anticancer effect strongly depended not only on the type, but also on the quantity of the secreted cytokine. The optimal cytokine concentrations were about $10,50,60$, and $30 \mathrm{ng} / 1 \times 10^{6}$ cells $/ 24 \mathrm{~h}$ for vaccines producing GM-CSF, IL-4, IL- $2,{ }^{8}$ or IL- $12,{ }^{8}$ respectively. In the case of IL-4-, IL-2-, ${ }^{8}$ or IL-12expressing $^{8}$ vaccines, the therapeutic effect increased at higher cytokine concentrations. In contrast, the antitumor effect of the GM-CSF-producing vaccine decreased at higher cytokine levels (Fig 2A). Most strikingly, the GMCSF vaccine produced at $200 \mathrm{MOI}$ ( $80 \mathrm{ng}$ GM-CSF/ $1 \times 10^{6}$ cells $/ 24 \mathrm{~h}$ ) was ineffective when applied alone. When the cytokine concentration secreted by the latter vaccine was lowered, by mixing GM-CSF-producing cells with nonsecreting cells in 1:3 ratios, the lower cytokine concentration resulted in a slightly improved anticancer effect. The increased tumor growth-inhibitory effect of the mixture was maintained when vaccination therapy was combined with radiation therapy (Fig 6B). The scientific background of the strong dependence on the cytokine concentration is not completely clear. It is well known, however, that a certain cytokine can influence a wide range of immune stimulatory mechanisms and usually different optimal concentrations are necessary for various target cells. ${ }^{34}$ Probably, only the activation of certain target cells will result in long-lasting antitumor immunity. It is also possible that supraoptimal doses may prove inhibitory for a certain action. ${ }^{34}$ We think that the strong dependence of the anticancer effect on the cytokine level might explain the very moderate success of clinical trials using autologous cancer cell vaccines for the treatment of human malignancies.

Wakimoto et $\mathrm{al}^{15}$ used a vaccine secreting both GM-CSF and IL-4 for the treatment of intracranial transplanted B16 melanoma and reported that the tumor growth-inhibitory effect was superior to a single cytokine-secreting vaccine. In the Gl261 mouse glioma model, we were unable to detect an increased survival for this combination. However, we cannot exclude that under suboptimal cytokine concentrations, the dual cytokine secretion is beneficial.

There are controversial data in the literature about the role of $\mathrm{CD}^{+}{ }^{+}$and $\mathrm{CD}^{+}$lymphocyte subsets in the anticancer effect of cytokine-modified cells. Several authors suggested that both cell types play an important role in the tumoricidal effect. ${ }^{15,17,18,23}$ Others support the preferential role of either $\mathrm{CD}^{+19}$ or $\mathrm{CD}^{+}$cells. ${ }^{16,26,27,29}$ According to our finding, both $\mathrm{CD}^{+}$and $\mathrm{CD}^{+}$cells are necessary for tumor eradication, at least for the GM-CSF-secreting vaccine. Pretreatment of brain tumor-bearing mice with monoclonal antibodies against either $\mathrm{CD}^{+}$or $\mathrm{CD}^{+}$lymphocytes completely abolished the antitumor effect of the GM-CSF vaccine (Fig 5 ). Moreover, when glioma-bearing mice were treated with GM-CSF-producing cells, the intracranial tumor was heavily infiltrated by $\mathrm{CD}^{+}$and moderately by $\mathrm{CD}^{+}$cells (Fig 4). We should mention that 10 days after treatment, the IL-2-, IL-4-, or IL-12-producing vaccines induced only $\mathrm{CD}^{+}{ }^{+}$infiltration at the tumor site. It is possible that $\mathrm{CD}^{+}$infiltration could be detected at other time points for these vaccines, as well.

The most important finding of our work is that local radiation therapy might be very efficiently combined by vaccination with cytokine-expressing autologous cancer cells for the treatment of experimental brain tumors. Recently, some other investigators also suggested that the combination of gene therapy with tumor irradiation might be beneficial. The intratumoral administration of a secretable angiostatin-like molecule into glioma xenografts by adenoviral injection had only marginal influence on tumor growth alone. However, its combination with radiation therapy resulted in a synergistic effect probably through the inhibition of tumor vascularization. ${ }^{35}$ Others detected the enhanced radiosensitivity of glioma cells after transduction with wild-type p53 encoding vectors. ${ }^{36-38}$ The radiosensitising effect of p53 is probably established through its apoptotic effect and p53 might also suppress tumor vascularization.

$\mathrm{Li}$ et $\mathrm{al}^{39}$ and Staba et $\mathrm{al}^{40}$ reported that the combination of radiation with intratumoral administration of a cytokine ( TNF $-\alpha$ )-encoding vector substantially slowed down tumor progression. We have found that cytokine-expressing vaccines might cure only $30-40 \%$ of brain tumor-bearing mice. Local radiation therapy alone hardly increased lifespans. However, the combination of these two modalities improved survival rates up to $80-100 \%$ (Fig 6). One simple explanation for the synergistic effect of vaccination and radiation therapies is that there is a continuous competition between tumor growth and tumor eradication by the activated immune system. Local irradiation decreases the tumor burden, so the activated immune system could overcome the decreased tumor mass. Another possibility is that after irradiation, the primary tumor cells die by necrosis. The necrotic death might lead to the liberation of immunogenic molecules that further enhances immune response.

The presented data indicated that the secreted cytokine level strongly influenced the antitumor effect of the cancer cell vaccines. Therefore, we suggest that future clinical trials should focus on the determination of the optimal cytokine concentrations by measuring the activation of the immune system. It is also possible that cytokine levels should be adjusted to the individual needs of the cancer patient. Our most important finding is that vaccination with cytokine-expressing cancer cells could be combined successfully with local irradiation of the tumor even under suboptimal therapeutic conditions. This suggests that vaccination therapy might open a new potential on the clinical treatment of gliomas when applied as an adjuvant treatment to existing therapeutic modalities, namely to surgery and radiation therapy, to eradicate residual tumor cells. 


\section{Acknowledgments}

This work was supported by an international collaborative ICGEB (CRP/HUN98-02-t2) Grant and Hungarian National Founds: ETT 6100/1/2000, ETT 247/1996, OTKA T-025810, OTKA T-032499, OMFB 96-97-651201 to GS, and OTKA T-025333, AKP-96/2-654-3.2 to EH. The authors thank Maria Frigyesi and Erzsebet Fekete for technical assistance.

\section{References}

1. Shoenberg BS. In: Walker MD, ed. Oncology of the Nervous System. Boston: Martinus Nijhoff; 1983:1-30.

2. Mahaley MS, Mettlin C, Natarajan N, et al. National survey of patterns of care for brain-tumor patients. J Neurosurg. 1989;71:826-836.

3. Shawler DL, Fakhrai H, Van Beveren C, et al. Gene therapy approaches to enhance antitumor immunity. Adv Pharmacol. 1997;40:309-37.

4. Forni G, Foa R, Santoni A, Frati L, eds. Cytokine-Induced Tumor Immunogenicity. San Diego, CA, USA: Academic Press; 1994.

5. Dranoff G, Jaffee E, Lazenby A, et al. Vaccination with irradiated tumor cells engineered to secrete murine granulocyte-macrophage colony-stimulating factor stimulates potent, specific, and long-lasting anti-tumor immunity. Proc Natl Acad Sci USA. 1993;90:3539-3543.

6. Allione A, Consalvo $\mathrm{M}$, Nanni $\mathrm{P}$, et al. Immunizing and curative potential of replicating and nonreplicating murine mammary adenocarcinoma cells engineered with interleukin (IL) -2, IL-4, IL-6, IL-7, IL-10, tumor necrosis factor alpha, granulocyte-macrophage colony-stimulating factor, and gamma-interferon gene or admixed with conventional adjuvants. Cancer Res. 1994;54:6022-6026.

7. Inoue M, Plautz GE, Shu S. Treatment of intracranial tumors by systemic transfer of superantigen-activated tumor-draining lymph node T cells. Cancer Res. 1996;56: 4702-4708.

8. Désaknai Sz, Lumniczky K, Hidvégi EJ, et al. Brain tumor treatment with IL-2 and IL-12 producing autologous cancer cell vaccines. Adv Exp Med Biol. 2001;495:369-372.

9. Shapiro WR, Ausman JI, Rall DP. Studies on the chemotherapy of experimental brain tumors: evaluation of 1,3-bis(2chloroethyl)-1-nitrosourea, cyclophosphamide, mithramycin and methotrexate. Cancer Res. 1970;30:2401-2413.

10. Nakamura Y, Wakimoto $\mathrm{H}$, Abe J, et al. Adoptive immunotherapy with murine tumor-specific $\mathrm{T}$ lymphocytes engineered to secrete interleukin 2. Cancer Res. 1994;54: 5757-5760.

11. Coligen JE, Kruisbeek AM, Marguiles DH, et al, eds. Current Protocols in Immunology. USA: Wiley; 1996:2.6.1-2.8.1.

12. Nakayama E, Shiku H, Takahashi T, et al. Definition of a unique cell surface antigen of mouse leukemia RL1 by cell-mediated cytotoxicity. Proc Natl Acad Sci USA. 1979; 76:3486-3490.

13. Saito S, Bannerji R, Gansbacher B, et al. Immunotherapy of bladder cancer with cytokine gene-modified tumor vaccines. Cancer Res. 1994;54:3516-3520.

14. Parney IF, Hao C, Petruk KC. Glioma immunology and immunotherapy. Neurosurgery. 2000;46:778-792.

15. Wakimoto H, Abe J, Tsunoda R, et al. Intensified antitumor immunity by a cancer vaccine that produces granulocyte- macrophage colony-stimulating factor plus interleukin 4. Cancer Res. 1996;56:1828-1833.

16. Sampson JH, Archer GE, Ashley DM, et al. Subcutaneous vaccination with irradiated, cytokine-producing tumor cells stimulates $\mathrm{CD}^{+}$cell-mediated immunity against tumors located in the immunologically privileged central nervous system. Proc Natl Acad Sci USA. 1996;93:10399-10404.

17. Yu JS, Burwick JA, Dranoff G, Breakefield XO. Gene therapy for metastatic brain tumors by vaccination with granulocytemacrophage colony-stimulating factor-transduced tumor cells. Hum Gene Ther. 1997;8:1065-1072.

18. Benedetti S, Bruzzone MG, Pollo B, et al. Eradication of rat malignant gliomas by retroviral-mediated, in vivo delivery of the interleukin 4 gene. Cancer Res. 1999;59: $645-652$.

19. Giezeman KM, Okada H, Brisette-Storkus CS, et al. Cytokine gene therapy of gliomas: induction of reactive $\mathrm{CD}^{+}{ }^{+} \mathrm{T}$ cells by interleukin-4-transfected 9L gliosarcoma is essential for protective immunity. Cancer Res. 2000;60: 2449-2457.

20. Chen B, Timiryasova TM, Andres ML, et al. Evaluation of combined vaccinia virus-mediated antitumor gene therapy with p53, IL-2, and IL-12 in a glioma model. Cancer Gene Ther. 2000;7:1437-1447.

21. Chen B, Timiryasova TM, Haghighat $P$, et al. Low-dose vaccinia virus-mediated cytokine gene therapy of gliomas. J Immunother. 2001;24:46-57.

22. Saleh M, Jonas NK, Wiegmans A, Stylli SS. The treatment of established intracranial tumors by in situ retroviral IFN-gamma transfer. Gene Ther. 2000;7:1715-1724.

23. Iwadate $\mathrm{Y}$, Tagawa $\mathrm{M}$, Namba $\mathrm{H}$, et al. Immunological responsiveness to interleukin-2-producing brain tumors can be restored by concurrent subcutaneous transplantation of the same tumors. Cancer Gene Ther. 2000;7:1263-1269.

24. DiMeco F, Rhines LD, Hanes J, et al. Paracrine delivery of IL12 against intracranial 9L gliosarcoma in rats. J Neurosurg. 2000;92:419-427.

25. Okada H, Giezeman-Smits KM, Tahara H, et al. Effective cytokine gene therapy against an intracranial glioma using a retrovirally transduced IL-4 plus HSVtk tumor vaccine. Gene Ther. 1999;6:219-226.

26. Saleh M, Wiegmans A, Malone Q, et al. Effect of in situ retroviral interleukin-4 transfer on established intracranial tumors. J Natl Cancer Inst. 1999;91:438-445.

27. Aoki T, Tashiro K, Miyatake SI, et al. Expression of murine interleukin 7 in a murine glioma cell line results in reduced tumorigenicity in vivo. Proc Natl Acad Sci USA. 1992;89:3850-3854.

28. Herrlinger U, Kramm CM, Johnston KM, et al. Vaccination for experimental gliomas using GM-CSF-transduced glioma cells. Cancer Gene Ther. 1997;4:345-352.

29. Natsume A, Tsujimura K, Mizuno M, et al. IFN-beta gene therapy induces systemic antitumor immunity against malignant glioma. J Neuro-Oncol. 2000;47:117-124.

30. Herrlinger U, Jacobs A, Quinones A, et al. Helper virus-free herpes simplex virus type 1 amplicon vectors for granulocyte-macrophage colony-stimulating factor-enhanced vaccination therapy for experimental glioma. Hum Gene Ther. 2000;11:1429-1438.

31. Glick RP, Lichtor T, de Zoeten E, et al. Prolongation of survival of mice with glioma treated with semiallogeneic fibroblasts secreting interleukin-2. Neurosurgery. 1999;45:867-874.

32. Natsume A, Mizuno M, Ryuke Y, Yoshida J. Antitumor effect and cellular immunity activation by murine interferon-beta gene transfer against intracerebral glioma in mouse. Gene Ther. 1999;6:1626-1633. 
33. Donson AM, Foreman NK. Adenovirus mediated gene therapy in a glioblastoma vaccine model; specific antitumor immunity and abrogation of immunosuppression. J Neuro-Oncol. 1998;40:205-214.

34. Thomson AW, ed. The Cytokine Handbook. New York, NY: Academic Press; 1994.

35. Griscelli F, Li H, Cheong C, et al. Combined effects of radiotherapy and angiostatin gene therapy in glioma tumor model. Proc Natl Acad Sci USA. 2000;97:66986703.

36. Gridley DS, Andres ML, Li J, et al. Evaluation of radiation effects against C6 glioma in combination with vaccinia virusp53 gene therapy. Int J Oncol. 1998;13:1093-1098.
37. Broaddus WC, Liu Y, Steele GT, et al. Enhanced radiosensitivity of malignant glioma cells after adenoviral p53 transduction. J Neurosurg. 1999;91:997-1004.

38. Badie B, Goh CS, Klaver J, et al. Combined radiation and $\mathrm{p} 53$ gene therapy of malignant glioma cells. Cancer Gene Ther. 1999;6:155-162.

39. Li J, Andres ML, Fodor I, et al. Evaluation of pGL1-TNFalpha therapy in combination with radiation. Oncol Res. 1998; 10:379-387.

40. Staba MJ, Mauceri HJ, Kufe DW, et al. Adenoviral TNF alpha gene therapy and radiation damage tumor vasculature in a human malignant glioma xenograft. Gene Ther. 1998;5: $293-300$. 